\title{
Analyse pollinique, propriétés physico-chimiques et action antibactérienne des miels d'abeilles africanisées Apis mellifera et de Méliponinés du Brésil
}

\author{
M Cortopassi-Laurino, DS Gelli
}

Instituto Adolfo Lutz, Seção de Microbiologia Alimentar, 01255 São Paulo, Brasil

(Reçu le 12 juillet 1989; accepté le 9 octobre 1990)

\begin{abstract}
Résumé - Nous avons analysé des miels d' Apis mellifera africanisée et d'abeilles sans aiguillon du Brésil. Les taux d'humidité et d'acidité libre sont plus faibles dans les miels d'Apis mellifera tandis que le pH montre des valeurs moindres dans les miels des Méliponinés, la teneur en eau étant la variable qui différencie le mieux les 2 groupes. Quant à la couleur, les 2 groupes se situent entre l'ambre et l'ambre clair. Nous avons testé des miels sur 7 souches différentes de bactéries. Dans les 2 groupes, la bactérie la plus sensible fut Bacillus stearothermophilus et la plus résistante Escherichia coli, les miels de la tribu des Trigonini étant plus efficaces que ceux de la tribu des Meliponini. Classés en fonction de l'origine florale, les miels les plus actifs contre les bactéries sont ceux dans lesquels prédomine le pollen des genres Mimosa et Eucalyptus pour le groupe $\mathrm{G}_{1}$ des Apis mellife$\mathrm{ra}$, tandis que pour le groupe $G_{2}$ des Méliponines, ce sont les miels à pollen de Borreria/Mimosa élaborés par Plebeia sp et les miels à Mimosa bimucronata fournis par Melipona subnitida.
\end{abstract}

Apis mellifera / Meliponinae / miel / activité bactériostatique / origine botanique / propriété physico-chimique / Brésil

\section{INTRODUCTION}

De tout temps, le miel a été utilisé pour ses propriétés antiseptiques et cicatrisantes ainsi que dans le traitement des affections respiratoires, des maladies du tractus digestif et des troubles cardiaques (Stomfay-Stitz et Kominos, 1960, Popesković et Dakić, 1979).

L'activité antibactérienne des miels était bien connue quand Dold et al (1937) en firent la démonstration au laboratoire en même temps que pour d'autres substances naturelles, sur 17 souches différentes de bactéries, donnant le nom d'inhibine à la substance qui inhibait la croissance des bactéries.

Adcock (1962) fut le premier à établir une corrélation entre les propriétés antibactériennes et les teneurs en peroxyde du miel. White et al (1962), White et Subers (1963) ont démontré que l'inhi- 
bine, qui est thermolabile et photolabile, provenait de la production naturelle du peroxyde d'hydrogène par la glucoseoxydase du miel.

Lavie (1968) a signalé un autre groupe d'inhibines photolabiles et thermostables qui, extraites du miel au moyen de solvants organiques, manifestaient des propriétés antibactériennes. Également à partir du miel et possédant ces mêmes propriétés, une substance semblable au lysosyme a été isolée par Mohring et Messner (1968) et une autre substance appartenant au groupe des flavonoïdes a été extraite par Bogdanov (1983).

Selon Molan et Russel (1988), I'origine florale du miel serait responsable des activités antibactériennes dues aux substances autres que des peroxydes. Des miels d'origine florale différente possèdent des activités inhibitrices différentes sur diverses souches de bactéries (lalomiteanu et Daghie, 1973; Popesković et Dakić, 1979).

Les acides organiques non dissociés sont également utilisés pour leurs activités antimicrobiennes (Ingram et al, 1956; Macris, 1975) car très solubles dans les membranes des cellules (Cramer et Prestegard, 1977), ils doivent provoquer des altérations dans la perméabilité cellulaire et dans la phosphorylation oxydative au niveau du transport des électrons (Freese et al, 1973). Dans les miels d'A mellifera, Stinson et al (1960) ont ainsi identifié entre autres acides organiques, les acides gluconique, butyrique, acétique, formique, malique, citrique, etc.

Les comparaisons entre les miels d' $A$ mellifera et ceux des Méliponines ne sont pas fréquentes. Gonnet et al (1964) ont remarqué que les teneurs en eau, en inhibine et en acidité sont un peu plus élevées et le $\mathrm{pH}$ plus bas dans les miels des Méliponinés qui, à la différence des miels d' $A$ mellifera, sont dépourvus d'amylase. Al- meida et Gurgel (1976) ont comparé de façon superficielle les miels de Melipona scutellaris avec ceux d' $A$ mellifera.

Le but de ce travail a été de rechercher, dans des miels provenant de 2 familles d'abeilles sociales, Apis mellifera et Méliponinés, dans quelle mesure leur origine botanique et leurs caractéristiques physico-chimiques $(\mathrm{pH}$, acidité libre, couleur, teneur en eau) ont une influence sur leurs propriétés antibactériennes. II contribue aussi à caractériser des miels de Méliponinés, pour lesquels il n'existe aucune législation, mais qui sont utilisés en thérapeutique dans les collectivités rurales.

\section{MATÉRIEL ET MÉTHODES}

Le matériel utilisé comporte 34 échantillons de miels récoltés dans diverses régions du Brésil, dont 20 proviennent d'abeilles africanisées (Apis mellifera $L$ ) et 14 sont fournis par 6 espèces de Meliponinae.

Les abeilles africanisées résultent du métissage avec les races européennes, survenu à partir de 1957, quand des abeilles africaines Apis mellifera scutellata furent introduites au Brésil.

Parmi les abeilles indigènes, nous avons étudié les espèces suivantes:

- Tetragona clavipes, (abréviation dans le texte: Tc) Selon Bettoni (1911), il s'agit certainement de l'espèce la plus productive en miel, lequel est toujours plus ou moins acide, ce qui a été confirmé par Nogueira-Neto (1953);

- Tetragonisca angustula, (abréviation dans le texte : Ta). C'est une des Méliponinés les plus communes, qui se rencontre dans de nombreux pays américains. Son miel est très savoureux (Nogueira-Neto, 1953). C'est une abeille très bien adaptée puisqu'elle fait son nid dans n'importe quelle cavité de taille appropriée;

- Melipona subnitida, (abréviation : Ms). Son miel, produit à raison de 1-2 I/ an, est consommé par les populations locales;

- Melipona quadrifasciata, (abréviation : Mq). Dans l'état de Sao Paulo, c'est l'espèce la plus commune du genre et malgré sa coutume de vi- 
siter les excréments, elle fournit un miel très savoureux (Nogueira-Neto, 1953);

- Plebeia sp correspond à plusieurs espèces dont la classification est en cours d'étude, (abréviation : $P I$. Son miel est acide et peu apprécié (Nogueira-Neto, 1953);

- Melipona scutellaris, (abréviation : Msc). Son miel est très savoureux, et selon Mariano Filho (1910), fortement balsamique, et beaucoup plus riche en substances aromatiques que le miel d' $A$ mellifera.

L'identification des grains de pollen des miels a été effectuée par comparaison avec la collection de référence qui se trouve au Laboratoire d'étude des abeilles du département d'écologie générale de l'université de São Paulo (USP). Les pollens des miels ont été traités, comme les pollens de référence, par la méthode de l'acétolyse (Erdtman, 1960).

Nous avons déterminé la couleur du miel suivant Anonyme (1980) par son absorbance dans un spectrophotomètre dont le 0 était calibré avec de la glycérine pure, en exprimant les résultats par les valeurs réelles obtenues. Les mesures ont été réalisées en cellules de $1 \mathrm{~cm}$ avec une longueur d'ondes de $560 \mathrm{~nm}$.

La teneur en eau et les substances solides solubles ont été déterminées au moyen d'un réfractomètre avec une variation de 0 à 90 . Nous avons mesuré le $\mathrm{pH}$ d'un échantillon de10 $\mathrm{g}$ de miel dilué dans $75 \mathrm{ml}$ d'eau déminéralisée.

Le taux d'acidité a été obtenu selon les mêmes techniques officielles en neutralisant jusqu'au $\mathrm{pH} 8,3$, par une solution d'hydroxyde de sodium $0,1 \mathrm{~N}$, des acides libres et les ions h:drogène libérés par les acides non dissociés durant le titrage.

Pour tester l'efficacité antibactérienne des miels, nous avons utilisé des souches de bactéries en culture de $24 \mathrm{~h}$, étalonnées pour la détection des substances antimicrobiennes (antibiologiques et antiseptiques) dans les aliments (Gelli et al, 1984). Ces souches de bactéries, conservées par lyophilisation et obtenues à partir de la collection de cultures de l'institut Adolfo Lutz de São Paulo qui provient elle-même de l'American type culture collection (ATCC) ou de l'Institut Pasteur (IP), sont les suivantes: Staphylococcus aureus ATCC 6538, Escherichia coli ATCC 0113/3, Klebsiella pneumomiae ATCC 10031, Pseudomonas aeruginosa ATCC 14502, Bacillus subtilis ATCC 6633 , Bacillus stearothermophilus variété calidolactis et Bacillus subtilis IP BA 155 (connue sous le nom de souche Caron).

Pour évaluer la capacité antibactérienne des miels, nous avons utilisé la méthode de Anonyme (1977), où l'application respectivement de $25,20,15,10$ et $5 \%$ de miel permet de graduer les résultats de 1 à 5 : Les résultats partiels $(0,25,0,5$ et 0,75$)$ correspondent à l'inhibition respective des bactéries à la surface de ces miels en culture solide (agar + miel). Le test de capacité bactériostatique ou bactéricide est considéré comme positif quand des échantillons de bactéries placés en solution de $\mathrm{BH}$ à $37^{\circ} \mathrm{C}$ pendant $24 \mathrm{~h}$ ne se développent pas en présence d'une concentration de miel à $25 \%$. La solution de $\mathrm{BHI}$ se trouble en cas de croissance bactérienne alors qu'elle reste limpide si les bactéries sont éliminées. Dans le cas des solutions troubles de $\mathrm{BH}$, nous avons examiné sur lame le matériel correspondant en ce qui concerne la morphologie, les propriétés tinctoriales et la présence de spores.

Pour les 2 groupes de miels $G_{1}$ (miel d'abeilles africanisées) et $\mathrm{G}_{2}$ (miel de Méliponinés), les résultats ont fait l'objet d'un traitement statistique. En raison de la structure multivariée des données, nous avons choisi de comparer les moyennes des variables dignes d'intérêt dans les groupes $G_{1}$ et $G_{2}$ par l'analyse de variance multivariée, selon le modèle de Johnson et Wichern (1982).

\section{RÉSULTATS}

\section{Origine et analyse pollinique}

Des résultats des analyses palynologiques, (tableaux I et II) nous n'avons retenu que les pollens dominants et leur pourcentage respectif.

\section{Analyse physico-chimique}

Les résultats de l'analyse physicochimique des miels montrent que la couleur des miels est peu variable : elle se 
Tableau I. Origine géographique et analyse pollinique des miels d'abeille africanisée (Apis mellifera).
Miels
$\%$ du pollen dominant

\begin{aligned} & 1 Eucalyptus sp (95\%) - São Paulo \\ & 2 Eucalyptus sp (73\%) - São Paulo \\ & 3 Eucalyptus sp (98\%) - São Paulo \\ & 4 Baccharis type $(84,7 \%)$ - Paraná \\ & 5 Baccharis type $(67,3 \%)$ - Senecio type $(13,2 \%)$ - Paraná \\ & 6 Baccharis type $(66,3 \%)$ - Paraná \\ & 7 Peptadenia gonoacantha (47,1\%) - Ceará \\ & 8 Mimosa bimucronata (26,8\%), Borreria verticillata (26.5\%) - Ceará \\ & 9 Myroxylum type (31,6\%), Byrsonima type $(29.1 \%)$ - Pará \\ & 10 Eucalyptus sp, Citrus sp (rare), Gramineae - São Paulo \\ & 11 Centella biflora s 1 (72,4\%) - Paraná \\ & 12 Myrtaceae et Compositae (rare) - São Paulo \\ & 13 Mimosa taimbensis (30\%) - Nordeste \\ & 14 Eupatorium sp (30\%) - Paraná \\ & 15 Eucalyptus sp $(98,9 \%)$ - São Paulo \\ & 16 Ilex (30.7\%), Eucalyptus sp (26.8\%) - São Paulo \\ & 17 Eucalyptus sp $(78,5 \%)$ - São Paulo \\ & 18 Eucalyptus sp (71,9\%) - São Paulo \\ & 19 Citrus sp (75,5\%) - Saõ Paulo \\ & 20 Citrus sp (92,1\%) - São Paulo \\ & \hline\end{aligned}

situe entre 0,1 et $1,0 \mu \mathrm{m}$, (absorbance) avec prédominance entre 0,3 et 0,6 , intervalle qui correspond aux couleurs ambre et ambre clair pour les 2 groupes de miels (fig 1C). La teneur en eau des miels d' $A$ mellifera varie de 16 à $22 \%$, celle des miels de Méliponinés, de 18 à $30 \%$, avec un cas unique atteignant $36 \%$ (fig 1D).

Le $\mathrm{pH}$ des miels varie de 3,2 à 4,6 avec une moyenne de 3,9 pour les miels d' $A$ mellifera et de 3,7 pour ceux de Méliponinés; les miels d' $A$ mellifera montrent un $\mathrm{pH}$ prédominant de l'ordre de 3,7, alors que pour les Méliponinés, les valeurs prédominantes du $\mathrm{pH}$ se situent entre 3,6 et 3,8 (fig 1A).

Les taux d'acidité libre varient de 10 à $70 \mathrm{meq} / \mathrm{kg}$ pour les miels d'A mellifera avec prépondérance des valeurs situées entre 10 et $20 \mathrm{meq} / \mathrm{kg}$, tandis que ceux des miels de Méliponinés, sont compris entre 30 et $90 \mathrm{meq} / \mathrm{kg}, 5$ échantillons présentant une acidité très élevée avec des valeurs dépassant $160 \mathrm{meq} / \mathrm{kg}$ (fig 1B).

Pour les variables physico-chimiques (tableau III), des miels du groupe $G_{1}$, le cœfficient de variation montre de hautes valeurs en ce qui concerne la couleur $(65,4)$ et l'acidité libre $(29,0)$ alors que l'humidité $(8,9)$ et le $\mathrm{pH}(10,5)$ sont plus homogènes; dans le groupe $G_{2}$, le cœefficient de variation concernant la couleur $(60,8)$ et l'acidité libre $(67,3)$ révèle une grande hétérogénéité des mesures alors que celui $\mathrm{du} \mathrm{pH}(9,7)$ indique une faible variation. L'humidité est la variable qui permet le mieux de distinguer les 2 groupes de miels d'abeilles (tableau IV). 
Tableau II. Origine géographique et analyse pollinique des miels de Meliponinae.

Miels

$\%$ du pollen dominant

\begin{tabular}{|c|c|}
\hline $\begin{array}{l}M s c_{1} \\
M s c_{2}\end{array}$ & $\begin{array}{l}\text { Mimosa bimucronata }(34,7 \%) \text {, Borreria verticillata }(27,5 \%) \text { - Rio.Grande do Norte } \\
\text { Piptadenia type }(72,1 \%) \text { - Ceará }\end{array}$ \\
\hline $\mathrm{Msc}_{3}$ & $\begin{array}{l}\text { Borreria verticillata }(54,2 \%) \text {, Mimosa bimucronata }(21,8 \%) \text { - Ceará } \\
\text { Mimosa taimbensis s1 }(82,2 \%) \text { - Ceará }\end{array}$ \\
\hline$M^{M} c_{5}$ & Borreria latifolia $(45,7 \%)$, Mimosa bimucronata $(34,5 \%)$ à Paraíba \\
\hline $\begin{array}{l}M s_{1} \\
M s_{2}\end{array}$ & $\begin{array}{l}\text { Mimosa bimucronata }(82,8 \%) \text { - Paraiba } \\
\text { Mimosa bimucronata }(98,5 \%) \text { - Ceará } \\
\text { Borreria verticillata }(44 \%) \text { Mimosa bimucronata }(30,6 \%) \text { - Paraiba }\end{array}$ \\
\hline $\begin{array}{l}\mathrm{Ta}_{1} \\
\mathrm{Ta}_{2} \\
\mathrm{Ta} \\
\mathrm{Mq_{1 }} \\
\mathrm{Mq_{2 }} \\
\mathrm{Tc}\end{array}$ & $\begin{array}{l}\text { Centella type }(55,4 \%) \text {, Cassia type }(15,7 \%) \text { - Paraná } \\
\text { Eucalyptus sp }(64,0 \%) \text {, Myroxylum balsamum }(5,8 \%) \text { - São Paulo } \\
\text { Piptadenia type }(32,6 \%) \text { n Bougainvillea spectabile }(16,0 \%) \text { - São Paulo } \\
\text { Myrcia type }(46,0 \%) \text {, Cassia type }(22,5 \%) \text { - São Paulo } \\
\text { Eucalyptus sp }(88,5 \%) \text { - São Paulo } \\
\text { Caesalpinia peltophoroides }(36,9 \%) \text {, Datura type }(30,0 \%) \text { - Paraná }\end{array}$ \\
\hline
\end{tabular}

Msc = Melipona scutellaris $;$ Ms = Melipona subnitida; $P_{l}=$ Pleibeia $\mathrm{sp} ; T a=$ Tetragonisca angustula; $M q=$ Melipona quadrifasciata; $T c=$ Tetragona clavipes .

Tableau III. Analyse statistique des variables physico-chimiques et de la capacité antibactérienne des miels des groupe $G_{1}$ (abeille africanisée) et $G_{2}$ (Meliponinae). $N=$ nombre d'observations; $\bar{x}=$ moyenne; $C V=$ coefficient de variation; $D P=$ écart type; $E P=$ erreur type; $M d=$ médiane; $C O L=$ couleur; $H U M=$ teneur en eau; $A C L=$ acidité libre.

Bs BsC Sa $\begin{gathered}\text { Bactéries } \\ \text { Ba }\end{gathered}$

\begin{tabular}{|c|c|c|c|c|c|c|c|c|c|c|c|}
\hline \multicolumn{12}{|c|}{ Apis mellifera } \\
\hline$N$ & 20 & 20 & 20 & 20 & 20 & 20 & 20 & 19 & 20 & 20 & 20 \\
\hline $\bar{x}$ & 2,75 & 2,68 & 3,15 & 2,99 & 2,95 & 1,99 & 4,13 & 0,68 & 19,21 & 3,90 & 12,35 \\
\hline$D P$ & 0,7 & 0,62 & 0,92 & 0,94 & 0,57 & 0,76 & 0 , & 0,44 & 1,71 & 0,41 & 3,59 \\
\hline$M d$ & 3,00 & 2,75 & 3,13 & 3,00 & 3,00 & 2,00 & 4,00 & 0,55 & 18,75 & 3,75 & 12,00 \\
\hline $\operatorname{Cv}(\%)$ & 27,2 & 23,1 & 29,1 & 31,4 & 19,2 & 38,2 & 8,7 & 65,4 & 8,9 & 10,5 & 29, \\
\hline$E P$ & 0,17 & 0,14 & 0,21 & 0,21 & 0,13 & 0,17 & 0,08 & 0,10 & 0,38 & 0,09 & 0,80 \\
\hline
\end{tabular}

\section{Meliponinés}

$\begin{array}{lccccccccccc}N & 14 & 14 & 14 & 14 & 14 & 14 & 13 & 13 & 13 & 13 & 13 \\ \bar{X} & 3,70 & 3,71 & 3,66 & 3,86 & 3,80 & 3,14 & 4,71 & 0,74 & 25,12 & 3,71 & 14,31 \\ D P & 0,98 & 0,73 & 0,85 & 0,96 & 0,90 & 1,47 & 0,52 & 0,45 & 5,36 & 0,36 & 9,63 \\ M d & 3,75 & 4,00 & 3,88 & 3,50 & 3,75 & 3,25 & 5,00 & 0,58 & 24,80 & 3,70 & 11,0 \\ C V & 26,0 & 20,0 & 23,0 & 25,0 & 24,0 & 47,0 & 11,0 & 60,8 & 21,3 & 9,7 & 67,3 \\ E P & 0,26 & 0,20 & 0,23 & 0,26 & 0,24 & 0,39 & 0,14 & 0,12 & 1,49 & 0,10 & 2,67\end{array}$



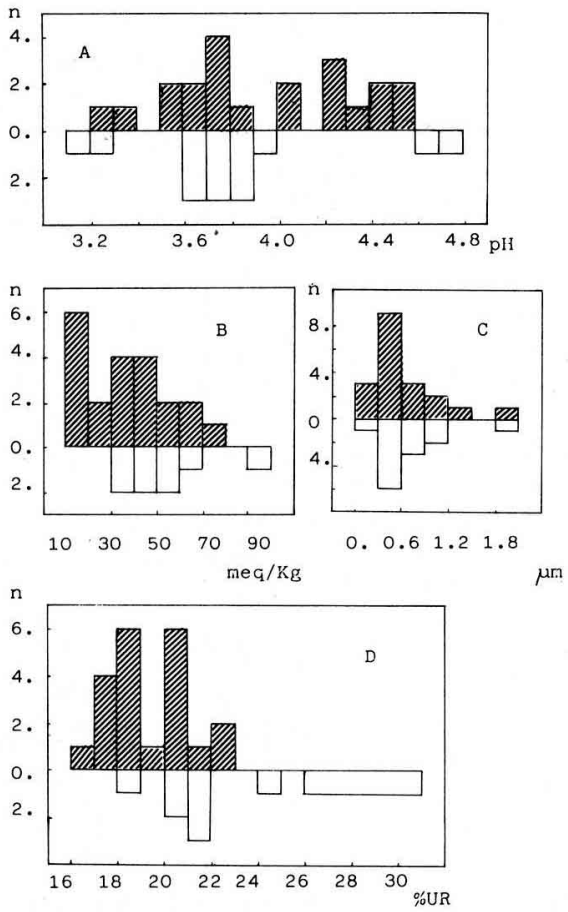

Fig 1. Variations des données physicochimiques des miels concernant les 2 groupes d'abeilles. $A=\mathrm{pH}, \mathrm{B}=$ acidité libre, $\mathrm{C}=$ couleur (absorbance), $\mathrm{D}=$ teneur en eau; $\mathbf{n}=$ nombre d'observations; mabeille africanisée; $\square$ Méliponinés.

\section{Action antibactérienne}

Les tableaux $V$ et VI présentent les données concernant l'activité antibactérienne des miels d' $A$ mellifera et des miels de Méliponinés respectivement. II en résulte que toutes les souches de bactéries ont été affectées par les différents miels examinés, leur degré d'inhibition variant en fonction du miel utilisé et de la souche bactérienne considérée.

L'activité inhibitrice des miels d'A mellifera se manifeste pour des concentrations comprises entre 12,5 et $20,5 \%$ (tableau V) qui correspondent à la graduation de 4,1
Tableau IV. Test de significativité des mesures de capacité antibactérienne, de couleur, de $\mathrm{pH}$, d'humidité et d'acidité libre entre les miels d'abeille africanisée et les miels de Meliponinae. ns $P>0,05 ;{ }^{*} P<0,05 ;{ }^{* *} P<0,01$.

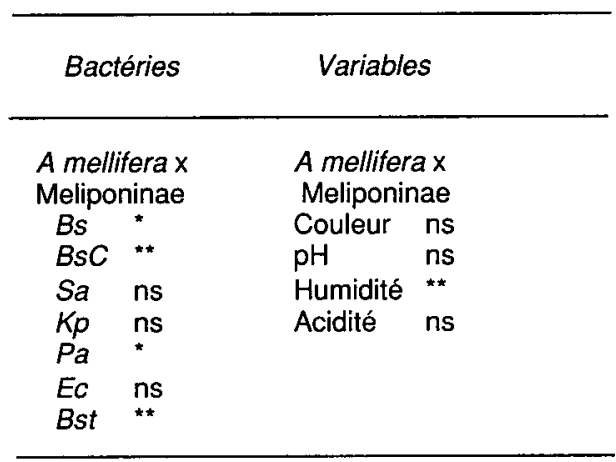

et 1,9 respectivement avec une moyenne égale à $15 \%$.

Celle des miels de Méliponinés se situe entre 5,5 et $17,5 \%$ (tableau VI) avec une moyenne de $11 \%$. Chez ces dernières, les miels de $M s c$ se révèlent équivalents à ceux d'A mellifera; cependant comme nous avons testé les miels de diverses abeilles indigènes ces valeurs furent différentes selon les espèces. Ainsi, les 2 miels de $M s$ provoquèrent une inhibition des bactéries pour des concentrations comprises entre 6,5 et $13,5 \%$ alors que les 3 échantillons de $\mathrm{Ta}$ furent efficaces entre 8 , et $14,5 \%$, les 5 échantillons de $M s c$, entre $13 \%$ et $17,5 \%$ et les 2 échantillons de $M q$, entre 7,5 et $8 \%$. Les échantillons uniques de $T c$ et de $P /$ montrèrent une activité antibactérienne pour des concentrations moyennes de miel égales respectivement à $7 \%$ et à $5,5 \%$.

Les propriétés bactériostatiques sont prédominantes par rapport aux propriétés bactéricides, mais un même échantillon de miel peut présenter les 2 sortes de propriétés en fonction de la souche bactérienne utilisée. La comparaison des 2 groupes de miels indique que ceux des $A$ mellifera 
Tableau V. Activité antibactérienne, exprimée en graduations de 1 à 5 , des miels d'abeille africanisée. Bs : Bacillus subtilis (6-392.106); BsC = Bacillus subtilis Caron (26 000.106); Sa: Staphylococcus aureus (23-126.106); Kp: Klebsiella pneumoniae (6-111.106); $\mathrm{Pa}:$ Pseudomonas aeruginosa (1642210.106); Ec : Escherichia coli (1000-5000.106); Bst : Bacillus stearothermophilus (0.6-4.106).

\begin{tabular}{|c|c|c|c|c|c|c|c|c|}
\hline \multirow[b]{2}{*}{ Miels } & \multirow[b]{2}{*}{$B s$} & \multirow[b]{2}{*}{$B s C$} & \multicolumn{3}{|c|}{$\begin{array}{c}\text { Activité antibactérienne } \\
\text { Souches }\end{array}$} & \multirow[b]{2}{*}{$E c$} & \multirow[b]{2}{*}{$B s t$} & \multirow[b]{2}{*}{$\bar{x}^{\prime}$} \\
\hline & & & $S a$ & $K p$ & $\mathrm{~Pa}$ & & & \\
\hline 1 & 3,0 & 3,0 & 4,75 & 3,0 & 3,5 & 2,75 & 4,0 & 3,43 \\
\hline 2 & 3,0 & 2,75 & $4,0^{*}$ & $3,0^{*}$ & $2,75^{*}$ & $2,75^{\star}$ & 3,75 & 3,14 \\
\hline 3 & 3,0 & 2,5 & 3,75 & $3,0^{*}$ & $3,0^{*}$ & 2,75 & 3,75 & 3,11 \\
\hline 4 & 3,0 & 3,25 & 4,0 & $2,0^{\star}$ & $3,0^{\star}$ & 3,0 & 4,0 & 3,18 \\
\hline 5 & 3,75 & 3,0 & 3,0 & $3,0^{*}$ & $3,75^{\star}$ & 2,0 & 4,0 & 3,21 \\
\hline 6 & 2,50 & 2,25 & 3,5 & 1,0 & $2,75^{\star}$ & 1,5 & 4,0 & 2,5 \\
\hline 7 & 3,0 & 2,0 & 3,25 & $2,0^{*}$ & $2,5^{\star}$ & 2,25 & $4,0^{*}$ & 2,71 \\
\hline 8 & 3,25 & 3,25 & 4,0 & 2,0 & 3,75 & 2,0 & 5,0 & 3,32 \\
\hline 9 & 2,5 & 2,25 & 3,0 & 3,25 & $3,0^{\star}$ & 3,0 & 4,0 & 3,0 \\
\hline 10 & 4,0 & $3,0^{*}$ & 2,75 & $3,25^{*}$ & 3,75 & 2,0 & 4,0 & 3,25 \\
\hline 11 & 3,0 & 3,0 & 3,25 & $3,75^{\star}$ & $3,0^{*}$ & 1,0 & $4,0^{*}$ & 3,0 \\
\hline 12 & 3,0 & 3,5 & 3,75 & $4,25^{\star}$ & 3,0 & 2,0 & $4,0^{\star}$ & 3,21 \\
\hline 13 & 3,75 & 3,25 & 2,75 & $4,75^{\star}$ & 3,75 & 1,5 & $5,0^{*}$ & 3,54 \\
\hline 14 & 2,0 & 0,75 & $1,75^{\star}$ & $2,75^{*}$ & $2,0^{*}$ & 0,5 & $3,75^{*}$ & 1,93 \\
\hline 15 & 3,0 & 2,75 & 4,0 & $3,75^{\star}$ & $2,0^{*}$ & 2,75 & $4,0^{*}$ & 3,18 \\
\hline 16 & 2,75 & 2,5 & 3,75 & $2,25^{\star}$ & 2,0 & 2,75 & $4,5^{\star}$ & 2,93 \\
\hline 17 & 1,75 & 3,0 & 2,0 & $4,0^{*}$ & $3,0^{*}$ & 1,0 & $4,0^{*}$ & 2,68 \\
\hline 18 & 2,0 & 2,75 & 0,75 & $3,75^{\star}$ & $3,0^{*}$ & 1,0 & $4,25^{*}$ & 2,50 \\
\hline 19 & 1,0 & 2,0 & 3,0 & $3,25^{\star}$ & $3,0^{*}$ & 1,5 & $4,0^{\star}$ & 2,54 \\
\hline 20 & 1,75 & $2,75^{\star}$ & 3,0 & 1,75 & 2,5 & 1,75 & $4,5^{*}$ & 2,57 \\
\hline $\bar{x}$ & 2,75 & 2,68 & 3,15 & 2,99 & 3,05 & 1,99 & 4,13 & \\
\hline
\end{tabular}

* Miels bactéricides.

sont bactéricides dans $30,7 \%$ des tests et ceux des Méliponinés dans $40,8 \%$. La bactérie la plus sensible fut Bacillus stearothermophilus et la plus résistante, Escherichia coli.

Quand les bactéries sont classées par leur degré d'inhibition (fig 2), on constate que les souches suivantes sont plus sensibles aux miels des Méliponinés qu'aux miels d'A mellifera dans le même ordre; $B$ stearothermophilus, $P$ aeruginosa, $B$ subtilis 6633 et $E$ coli. Cependant, les souches de $B$ subtilis Caron, K pneumoniae et de $S$ aureus font exception, la dernière se situant complètement en dehors de cette séquence.
En divisant le degré d'inhibition bactérienne des miels des Méliponinés par celui des miels de Apis, on obtient, pour chaque souche, un pourcentage qui traduit l'accroissement de l'inhibition par les miels des Méliponinés : Bst (14\%), Sa (16\%), Pa $(24 \%), K p(29 \%)$, Bs (35\%), BsC (38\%) et Ec $(52 \%)$.

Dans le groupe des Méliponinés, les miels fournis par les Trigonini ( $M \mid C=9 \%$ ) ont montré une plus grande activité antibactérienne que ceux des Meliponini $(\mathrm{MIC}=12 \%)$.

L'analyse multivariée des 2 groupes de miels (tableau III) n'a pas montré d'obser- 
Tableau VI. Activité antibactérienne, exprimée en graduations de 1 à 5 , des miels de Meliponinae; souches testées : voir légende tableau V; miels testés : voir légende tableau II.

\begin{tabular}{|c|c|c|c|c|c|c|c|c|}
\hline \multirow[b]{2}{*}{ Miels } & \multicolumn{8}{|c|}{$\begin{array}{c}\text { Activité antibactérienne } \\
\text { Souches }\end{array}$} \\
\hline & Bs & $B s C$ & Sa & $K p$ & $\mathrm{~Pa}$ & $E c$ & $B s t$ & $\overline{x^{\prime}}$ \\
\hline $\mathrm{Msc}_{1}$ & 3,0 & 4,0 & 2,0 & $3,25^{*}$ & 2,5 & 1,5 & 5,0 & 3,04 \\
\hline $\mathrm{Msc}_{2}$ & 3,0 & 2,25 & 2,5 & 2,25 & 2,25 & 1,75 & 3,5 & 2,5 \\
\hline $\mathrm{Msc}_{3}$ & 3,5 & 3,5 & 3,0 & 3,0 & $3,75^{\star}$ & 2,0 & $5,0^{*}$ & 3,39 \\
\hline $\mathrm{Msc}_{4}$ & 2,0 & 3,0 & 3,0 & 3,5 & $3,25^{\star}$ & 1,0 & $4,0^{*}$ & 2,82 \\
\hline $\mathrm{Msc}_{5}$ & 3,25 & 3,5 & 3,75 & 3,5 & $3,25^{\star}$ & 2,0 & $4,75^{\star}$ & 3,43 \\
\hline $\mathrm{Ms}_{1}$ & 3,5 & 3,0 & 3,0 & 3,5 & $3,5^{\star}$ & 2,5 & $4,0^{*}$ & 3,29 \\
\hline $\mathrm{Ms}_{2}$ & 4,75 & 4,75 & 4,75 & 5,0 & $4,0^{*}$ & $5,0^{*}$ & $5,0^{*}$ & 4,75 \\
\hline$P_{1}$ & 5,0 & $5,0^{*}$ & $4,75^{\star}$ & 5,0 & $5,0^{*}$ & $5,0^{*}$ & $5,0^{*}$ & 4,96 \\
\hline $\mathrm{Ta}_{1}$ & 4,25 & 4,0 & $4,0^{*}$ & 3,0 & $3,75^{\star}$ & $4,0^{*}$ & $5,0^{*}$ & 4,0 \\
\hline $\mathrm{Ta}_{2}$ & 4,75 & 4,0 & $4,0^{*}$ & 4,0 & $4,75^{\star}$ & $4,25^{\star}$ & $5,0^{*}$ & 4,39 \\
\hline $\mathrm{Ta}_{3}$ & 2,0 & 3,0 & 3,75 & $3,0^{*}$ & 3,0 & $1,75^{\star}$ & $5,0^{*}$ & 3,07 \\
\hline $\mathrm{Mq}_{1}$ & 4,0 & $4,0^{*}$ & 4,75 & 5,0 & 4,75 & $4,0^{*}$ & - & 4,42 \\
\hline $\mathrm{Mq}_{2}$ & 4,0 & 4,0 & $4,0^{*}$ & 5,0 & 4,5 & $4,5^{\star}$ & $5,0^{*}$ & 4,43 \\
\hline $\mathrm{Tc}^{12}$ & 4,75 & 4,0 & $4,0^{*}$ & 5,0 & $5,0^{*}$ & $4,75^{\star}$ & $5,0^{*}$ & 4,64 \\
\hline $\bar{x}$ & 3,70 & 3,71 & 3,66 & 3,86 & 3,80 & 3,04 & 4,71 & \\
\hline
\end{tabular}

vation aberrante $(P>0,05)$. Dans les 2 groupes, c'est $B$ stearothermophilus (Bst) qui présente le plus faible écart type et le plus petit cœefficient de variation. Au

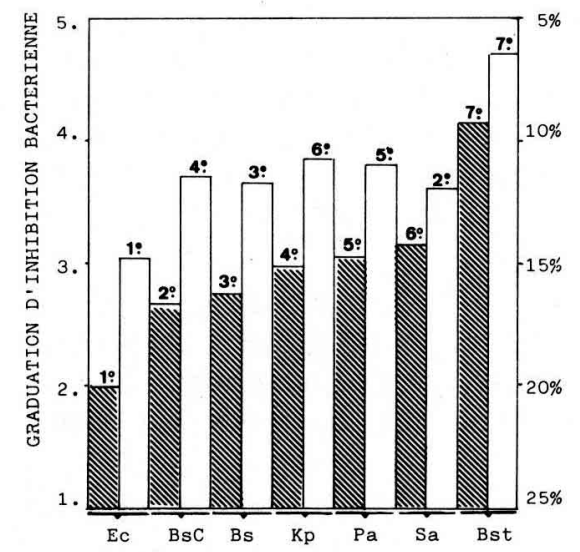

contraire, $K$ pneumoniae et $E$ coli manifestent la plus forte hétérogénéité des mesures puisque dans le $1^{\mathrm{er}}$ groupe, le cœfficient de variation est de 31,4 et de 38,2 respectivement, et dans le $2^{\mathrm{e}}$ groupe, il atteint la valeur de 47,0 pour $E$ coli.

\section{DISCUSSION}

Les données polliniques ont montré que les miels qui possèdent le plus large

Fig 2. Taux d'inhibition des échantillons bactériens par les miels d'Apis mellifera africanisée et des Méliponinés $\square$; Bs: Bacillus subtilis (6-392.106)*; BsC: Bacillus subtilis Caron $\left(26000.10^{6}\right)^{*}$; Sa: Staphylococcus aureus (23-126.106)*; Kp: Klebsiella pneumoniae $\left(6-111.10^{6}\right)^{*}$; Pa: Pseudomonas aeruginosa (164-2 210.106)*; Ec: Escherichia coli (1 000-5 000.106)*; Bst: Bacillus stearothermophilus $\left(0.6-4.10^{6}\right)^{*}$. * Nombre de bactéries viables par $\mathrm{ml}$ de bouillon. 
spectre antibactérien sont, dans le fer groupe (miels d'abeilles africanisées), ceux qui présentent une prédominance de pollen des genres Mimosa et Eucalyptus, et dans le $2^{\mathrm{e}}$ groupe (miel de Méliponinés), ceux à pollen de Borreria/Mimosa fournis par l'abeille $P$ l et ceux à pollen de Mimosa élaborés par l'abeille Ms. Par ailleurs, 2 échantillons de miel produits par $M q$ avec des origines florales différentes ont reçu pratiquement les mêmes notes de capacité antibactérienne; au contraire, 2 échantillons de miel produits par Msc avec la même origine florale ont reçu des notes différentes. Ces observations suggèrent donc que, pour cette espèce d'abeille il n'y a pas de relation entre l'origine florale et la capacité antibactérienne des miels. Des résultats plus cohérents ont été obtenus avec les miels de Msc dont l'origine florale était Borreria. D'autres recherches seront nécessaires pour établir des données plus spécifiques concernant les différentes souches de bactéries.

Chez $A$ mellifera, les miels monofloraux de Citrus ont montré, avec les divers tests réalisés, une uniformité de résultats concernant les propriétés antibactériennes et l'acidité libre qui ne se trouve pas dans les miels d'Eucalyptus. Cependant, les miels d'Eucalyptus provenant de 2 genres différents de Méliponinés, se sont comportés de manière semblable à l'égard de ces mêmes tests.

Pour Barbier et Pangaud (1961), la certitude de l'origine botanique d'un miel repose avant tout sur l'identification des pollens qu'il contient, mais la détermination de sa couleur et de sa courbe de neutralisation des acides libres apporte un complément nécessaire aux résultats de l'analyse pollinique.

En accord avec la législation, les taux d'acidité libre peuvent varier, dans les miels d'Apis, jusqu'à $40 \mathrm{meq} / \mathrm{kg}$. Cependant, de nombreux miels d'Apis et de Méli- poninés qui n'ont pas fait l'objet d'une analyse appropriée, dépassent certainement cette limite. Des taux élevés d'acidité peuvent signifier que le miel se trouve en fermentation et qu'il possède une odeur acide subtile ou même pénétrante (Crane, 1975; Crane et Walker, 1984). Gonnet (1965), Perez et Rodrigues (1970), Wootton et al (1976) ont ainsi signalé que des miels d'A mellifera possédaient un niveau d'acidité situé bien au-dessus de la limite permise. Pour Apis dorsata, Minh et al (1971) ont trouvé des valeurs atteignant $81,79 \mathrm{meq} /$ $\mathrm{kg}$.

Les taux élevés d'acidité libre de miels des Méliponinés, probablement associés à plus forte teneur en eau, ont fourni des valeurs supérieures à $160 \mathrm{meq} / \mathrm{kg}$. Gonnet et al (1964) ont relevé dans des miels d'abeilles indigènes du Brésil, des valeurs de 104,8 et $117,0 \mathrm{meq} / \mathrm{kg}$ chez $S$ postica et $M$ quadrifasciata respectivement. Or dans nos résultats, parmi les 5 échantillons de miel qui présentaient des taux élevés d'acidité, 4 ont manifesté une forte activité antibactérienne.

En ce qui concerne les valeurs du $\mathrm{pH}$, nos résultats se situent dans le cadre de la moyenne préétablie pour les miels d'Apis. Dans le cas des miels de Méliponinés, hormis l'exemple unique de l'espèce Tetragonisca angustula dont le $\mathrm{pH}$ moyen était de 4,1 (Iwama, 1977), il n'y a pas de données antérieures, les valeurs que nous avons obtenues sont en moyenne inférieures à celles des miels d' $A$ mellifera.

Les miels des Méliponinés sont certainement plus aqueux que ceux d' $A$ mellifera et contiennent jusqu'à $36,4 \%$ d'eau. Ceci se traduit par une plus grande possibilité de fermentation et correspond probablement à un équilibre avec les taux élevés d'acidité signalés précédemment. Dans les miels de Tetragonisca angustula, Iwama (1977) a relevé une moyenne de $28,2 \%$ d'eau alors que pour cette espèce, la moyenne de nos données fut de $26,3 \%$. 
Des miels brésiliens provenant d'A mellifera ont présenté une teneur en eau de l'ordre de 15-20\% (Flechtmann et al, 1963).

Le $\mathrm{pH}$ d'un aliment est l'un des principaux facteurs qui déterminent, pendant sa préparation et son stockage, la survie et la croissance des micro-organismes. Cependant, il est difficile de séparer le rôle du $\mathrm{pH}$ des autres facteurs qui sont eux-mêmes influencés par le $\mathrm{pH}$. Les microorganismes sont affectés par la concentration des ions $\mathrm{H}^{+}$, c'est-à-dire par le $\mathrm{pH}$, et par la concentration en acides faibles non dissociés, lesquels sont à leur tour influencés par le $\mathrm{pH}$.

Les miels d'A mellifera et de Méliponinés offrent un champ d'action antibactérien d'une certaine ampleur puisqu'il sont efficaces à des concentrations inférieures à $20 \%$.

En ce qui concerne l'influence de la dilution des miels sur les bactéries, Prica (1938) a établi que des concentrations de $20 \%$ de miel avaient de fortes propriétés bactéricides, ce qui fut confirmé par lalomiteanu et Daghie (1973). Par contre, White et Subers (1963) ainsi que James et al (1972) ont conclu que l'effet de miels à une concentration de $25 \%$ était plutôt bactériostatique que bactéricide. Mais dans nos expériences, l'examen de bactéries inhibées par du miel et développées en solution de $\mathrm{BHI}$ a révélé surtout des modifications dans la morphologie, les propriétés tinctoriales et la formation des spores, ce qui suggère des altérations profondes dans la constitution cellulaire plutôt que dans la vitalité des bactéries.

La souche bactérienne la plus utilisée pour tester les propriétés antibactériennes des miels d'A mellifera a été $S$ aureus (Dold et al, 1938; Prica, 1938; White et al, 1962; Wooton et al, 1978; Yatsunami et Echigo, 1984; Bogdanov et al, 1987).
D'après nos résultats, ce n'est pas la souche la plus appropriée pour les miels car elle s'est montrée l'une des moins résistantes aux miels testées dans le groupe des abeilles. Le $B$ subtilis Caron, souche conforme à la législation pour l'expertise des miels en France, s'est révélé l'un des plus résistants aux miels d' $A$ mellifera, mais il fut aussi l'un des plus affectés par les miels de Méliponinés. Ces données suggèrent qu'il faut utiliser des souches différentes de bactéries pour tester des miels provenant d'espèces différentes d'abeilles. Pour Popeskovic et Dakić (1979), c'est $E$ coli qui représente la bactérie la plus résistante parmi les 10 souches considérées.

En ce qui concerne $S$ aureus, nos résultats confirment la coutume populaire des brésiliens qui utilisent les miels d'abeilles indigènes comme mesure préventive contre les infections oculaires provoquées par cette bactérie.

Les raisons de l'activité antibactérienne du miel sont matière à controverse. L'une des interprétations admises actuellement est celle de White et Subers (1963) selon laquelle le bioxyde d'hydrogène qui provient de la glucose-oxydase du miel, serait la substance inhibitrice des bactéries. Cependant, il est connu que le miel lui-même et les bactéries produisent de la catalase qui élimine le bioxyde d'hydrogène et que la production de catalase par les bactéries varie en fonction de leur cycle de développement (McCanthy et Hinshelwood, 1959). Yatsunami et Echigo (1984) suggèrent que cette activité du miel est associée à une haute concentration en sucres, à de basses valeurs du $\mathrm{pH}$ et à l'accumulation du bioxyde d'hydrogène. La théorie de l'inhibition des bactéries par des substances autres que des péroxydes a fait récemment plusieurs adeptes : lalomiteanu et Daghie, 1973; Popesdović et Dakić, 1979; Molan et Russell 1988. 
Pour chaque variable que nous avons déterminée, l'analyse statistique a mis en évidence une grande fluctuation des mesures. Certains indices suggèrent que la capacité antibactérienne des miels d'A mellifera est différente de celle des miels de Méliponinés pour Bacillus subtilis, Bacillus subtilis Caron, Pseudomonas aeruginosa et Bacillus stearothermophilus.

\section{REMERCIEMENTS}

Nous remercions vivement de leur aimable collaboration : MO Marmo, M Roger, J Lavallard, M Ari, D Amaral, Mme VL Imperatriz-Fonseca, Mme LK Cordani, et Mme E Brás.

Ce travail a reçu une aide substantielle du CNPq (150602-86 et 303455-87).

Summary - Pollen analysis, physicochemical properties and antibacterial action of Brazilian honeys from Africanized honeybees (Apis mellifera $L$ ) and stingless bees. Two groups of Brazilian honeys were analyzed :

- from Africanized honeybees (20 samples; = $\mathrm{G}_{1}$ );

- from stingless bees ( 14 samples; $=G_{2}$ ). In the palynological studies, the pollen grains were identified by comparing them with a reference catalogue at the University of São Paulo bee laboratory of the USP. The acetolysis method was used to facilitate observations. In $G_{1}$ the most active honeys against bacteria were those where Mimosa and Eucalyptus pollen was predominant. In $\mathrm{G}_{2}$, a honey produced by Plebeia sp with Borreria/Mimosa pollen and another by Melipona subnitida with Mimosa bimucronata pollen, were found to be most active against bacteria. There was less moisture and free acidity in the Apis honeys whereas the $\mathrm{pH}$ was lower in the honeys from stingless bees. Moisture was the characteristic which best differentiated the two groups (table IV). In colour, both ranged between amber and light-amber.

All honeys were assayed against 7 strains of bacteria (tables $\mathrm{V}$ et $\mathrm{VI}$ ). The honey was mixed in a concentration of 5$25 \%$ with agar and put on a plate; drops of bacterium culture were then spread inside this solid culture. After $24 \mathrm{~h}$ at $37^{\circ} \mathrm{C}$ a $1-5$ grading was given to the honey tested, depending on the bacterial growth. Honeys from stingless bees had a stronger inhibition capacity than Apis honeys but this varied according to the species of stingless bee. The honeys from Apis showed inhibition with an average concentration of $15 \%$, whereas in honeys from stingless bees the average inhibitory concentration was $11 \%$. The minimum concentrations were $12.5 \%$ and $5.5 \%$ respectively. For both groups the least resistant bacterium was Bacillus stearothermophilus (Bst) and the most resistant Escherichia coli (EC) (fig 2). Both groups of honeys were mainly bacteriostatic, ie in spite of sometimes damaging the bacteria they inhibited but did not kill them (Gram test). Honeys from the tribe Trigonini were found to be better antibacterial agents than those from Meliponini.

Apis mellifera / Meliponinae / honey / bacteriostatic activity / botanical origin / physico-chemical characteristic / Brazil

\section{Zusammenfassung - Pollenanalyse, physikochemische Eigenschaften und antibakterielle Wirkung des Honigs von Afrikanisierter Apis mellifera und von Stachellosen Bienen in Brasilien. Brasi- lianische Honige von Afrikanisierten Bie- nen (Apis mellifera; 20 Proben) und von Stachellosen Bienen (14 Proben) wurden analysiert. Bei der palynologischen Unter- suchung wurden die Pollenkörner durch Vergleich mit dem Referenzkatalog am Bienenlaboratorium der Universität São Paulo (USP) bestimmt. Es wurde das}


Azetolyse-Verfahren benutzt, welches die Pollenbeobachtung erleichtert. Die aktivsten Honige waren bei den Apis-Honigen diejenigen mit einem Überwiegen des Pollens von Mimosa und Eucalyptus, während es bei den Meliponen Borreria und Mimosa waren, eingetragen von Plebeia $\mathrm{sp}$, und ein Honig von Mimosa bimucronata gesammelt von Melipona subnitida.

Insgesamt waren bei den Apis-Honigen der Wassergehalt und der Gehalt an freien Säuren niedriger, der $\mathrm{pH}$ aber höher als bei den Honigen der Stachellosen Bienen. Der Wassergehalt ist ein variables Merkmal, das beide Gruppen trennen kann (Tabelle IV). Was die Farbstufen betrifft, so lagen beide zwischen bernstein- und hell-bernsteinfarben. Alle Honige wurden gegen sieben Bakterienstämme geprüft (Tabelle V und VI). Der Honig wurde in Konzentrationen von 5-25\% mit Agar gemischt und auf Platten ausgebracht; dann wurden Tropfen der Bakterienkulturen in diesem festen Nährboden verteilt. Nach 24 Stunden bei $37^{\circ} \mathrm{C}$ wurden die Kulturen je nach dem Wachstum der Bakterien in die Klassen 0-5 eingeteilt.

Als Ergebnis ist festzustellen, daß die Honige der Stachellosen Bienen eine stärkere Hemmwirkung auf die Bakterien hatten als diejenigen der Honigbienen, und $d a ß$ es auch zwischen den Arten der Meliponen Unterschiede gab. Die ApisHonige zeigten durchschnittliche Hemmwirkungen bei einer Konzentration von $15 \%$, die Meliponen-Honige bei einer Konzentration von $11 \%$. Die minimalen Konzentrationen betrugen $12,5 \%$, bzw $5,5 \%$. Bei beiden Honiggruppen war das empfindlichste Bakterium Bacillus stearothermophilus (Bst) und das resistenteste Escherichia coli (Ec) (Abb 2). Beide Gruppen wirkten in erster Linie bakteriostatisch, dh sie hemmen die Bakterien, töten sie aber nicht; allerdings werden die Bakterien manchmal doch geschädigt (Gram-Test).
Die Honige von dem Tribus Trigonini hatten eine bessere antibakterielle Wirkung als diejenigen von Meliponini.

\section{Apis mellifera / Meliponinae / Honig / antibakterielle Wirkung / physiko- chemische Eigenschaft / Brasilien / botanische Herkuntt}

\section{REFERENCES}

Adcock D (1962) The effect of catalase on inhibine and peroxide values of various honeys. $J$ Apic Res 1, 38-40

Almeida MG de, Gurgel CRB (1976) Análise comparativa entre o mel de Apis mellifera e o mel de Melipona scutellaris. Ciência e Cultura (Supl), 806

Anonyme (1977) Méthodes officielles d'analyse du miel. Journal Officiel de la République française, Paris, 22 avril, 3485-3514

Anonyme (1980) Técnicas de análise do mel de Abelhas. Jornal Oficial do Brasil. Brasilia, 28 março, 5568-5572

Barbier EC, Pangaud CY (1961) Origine botanique et caractéristiques physicochimiques des miel. Ann Abeille 4, 51-65

Bertoni AW (1911) Contribuicion a la biologia de las avispas y abejas del Paraguay. An Mus Nac Hist Nat Buenos Aires 3, 99

Bogdanov S (1983) Characterisation of antibacterial substances in honey. Lebensm Wiss Technol 17, 74-76

Bogdanov S, Rieder K, Rüegg M (1987) Neue Qualitätskriterian bei Honiguntersuchungen. Apidologie 18, 267-278

Cramer JA, Prestegard JH (1977) NMR studies of $\mathrm{pH}$ induces transport of carboxylic acids across phospholipod vesicle membranes. Biophys Res Commun 75, 295-301

Crane E (1975) Honey : A Comprehensive Survey. Heinemann, Londres, $608 p$

Crane E, Walker $P$ (1984) Composition of honeys from some important honey sources. Bee World 65, 167-174

Dold H, Du OH, Dziao ST (1937) Nachweis antibakterieller, itze und lichtempfindlicher Hem- 
mungsstoffe (Inhibine) im Naturhonig (Blütenhonig). Z Hyg Infektionskr 120, 155-167

Erdtman G (1960) The acetolysis method. Sven Bot Tidskr 54, 561-564

Flechtmann CHW, Caldas Filho CF, Amaral E, Arzola JD (1963) Análise de méis do Estado de São Paulo. Bol Ind Animal 21, 65-73

Freese E, Sheu CW, Galliers E (1973) Function of lipophilic acids as antimicrobial food additive. Nature (Londres), 241, 321-325

Gelli DS, Jakabi M, Souza A (1984) Inibidores Microbianos em leite pasteurizado do comércio da cidade de São Paulo. Rev Inst Adolfo Lutz 44, 19-24

Gonnet M (1965) Les modifications de la composition chimique des miels au cours de la conservation. Ann Abeille 8, 129-146

Gonnet M, Lavie P, Nogueira-Neto P (1964) Etude de quelques caractéristiques des miels récoltés par certains Méliponines brésiliens. CR Séances Acad Sci Paris, 258, 3107-3109

Ialomiteaunu, M, Daghie V (1973) Investigations of the antibiotic qualities of honey. XXIV Int Congr Apic Proc Buenos Aires 438-440

Ingram M, Ottoway FJH, Coppock JBM (1956) The preservative action of acid substances in food. Chem Ind (Lond) 42, 1154-1163

Iwama S (1977) Coleta de alimentos e qualidade do mel de Tetragonisca angustula angustula Latreille (Apidae, Meliponinae). Dissertação (Mestrado). Departamento de Zoologia, São Paulo, USP 135 p

James OBO'L, Segree W, Ventura AK (1972) Some antibacterial properties of Jamaican honey. WI Med J 12, 7-17

Johnson RA, Wichern DW (1982) Applied Multivariate Statistical Analysis. Englewood Cliffs, Prentice Hall Inc, $594 \mathrm{p}$

Lavie $P$ (1968) Les substances antibiotiques dans la colonie d'abeilles. In: Traité de biologie de l'abeille (Chauvin R, ed), Masson, Paris, t 3, 2-115

Macris BJ (1975) Mechanisms of benzoic acid uptake by Saccaromyces cerevisiæ. Appl Microbiol 30, 503-506

Mariano-Filho J (1910) Sobre os meios de defesa das abelhas sem ferrāo. Chácaras Quintals 12, 50-53
McCanthy BJ, Hinshelwood CFRS (1959) Variations in catalase activity during a bacterial growth cycle. Proc R Soc B 150, 13-23

Minh HV, Mendoza BV, Laigo FM (1971) The chemical composition of honey produced by Apis dorsata. J Apic Res 10, 91-97

Mohring W, Messner B (1968) Lysozym als antibakterielles Agens im Bienenhonig und Bienengift. Acta Biol Med Ger 21, 85-95

Molan PC, Russell KM (1988) Non-peroxide antibacterial activity in some New Zealand honeys. J Apic Res 27, 62-67

Nogueira-Neto $P$ (1953) A criação de abelhas indigenas sem ferrâo. Chácaras Quintas, São Paulo, 365 p

Perez BS, Rodregues AT (1970) Composition quimica y espectro polinico de mieles Españolas. An Bromat 22, 377-406

Popesković D, Dakić M (1979) Antibacterial effect of three honey types after heat treatment. XXVII Int Congr Apic, Athens, 491-495

Prica M (1938) Über die baktericide Wirkung des Naturhonigs. Z Hyg Infektionskr 120, 437-443

Stinson EE, Subers MH, Petty J, White,JW Jr (1960) The composition of honey. V. Separation and identification of the organic acids. Arch Biochem Biophys 89, 6-12

Stomfay-Stitz J, Kominos DD (1960) Über bakteriostatische Werkung des Honigs. $Z$ Lebensm Unters Forsch 113, 304-309

White JW Jr, Subers MH, Shepartz Al (1962) The identification of inhibine. Am Bee J 102, 430-431

White JW Jr, Subers MH (1963) Studies on honey inhibine. 2. A chemical assay. $J$ Apic Res 2, 93-100

Wootton M, Edwards RA, Faraji-Haremi R, Johnson AT (1976) Effect of accelerated storage conditions on the chemical composition and properties of Australian honeys. 1. Colour, acidity and total nitrogen content. J Apic Res 15, 23-28

Wootton M, Edwards RA, Rowse A (1978) Antibacterial properties of some Australian honeys. Food Technol Aust May, 175-178

Yatsunami K, Echigo T (1984) Antibacterial action of honey and royal jelly. Honeybee Sci 5 , 125-130 (in Japanese) 